ARTICLE

\title{
Ligand assisted growth of perovskite single crystals with low defect density
}

Ye Liu ${ }^{1,2}$, Xiaopeng Zheng ${ }^{2}$, Yanjun Fang ${ }^{2}$, Ying Zhou ${ }^{1}$, Zhenyi $\mathrm{Ni}^{1}$, Xun Xiao (1) ${ }^{1}$, Shangshang Chen (1) ${ }^{1} \&$ Jinsong Huang (i) ${ }^{1 凶}$

A low defect density in metal halide perovskite single crystals is critical to achieve high performance optoelectronic devices. Here we show the reduction of defect density in perovskite single crystals grown by a ligand-assisted solution process with 3-(decyldimethylammonio)-propane-sulfonate inner salt (DPSI) as an additive. DPSI ligands anchoring with lead ions on perovskite crystal surfaces not only suppress nucleation in solution, but also regulate the addition of proper ions to the growing surface, which greatly enhances the crystal quality. The grown $\mathrm{CH}_{3} \mathrm{NH}_{3} \mathrm{Pbl}_{3}$ crystals show better crystallinity and a 23-fold smaller trap density of $7 \times 10^{10} \mathrm{~cm}^{-3}$ than the optimized control crystals. The enhanced material properties result in significantly suppressed ion migration and superior $\mathrm{X}$-ray detection sensitivity of $\mathrm{CH}_{3} \mathrm{NH}_{3} \mathrm{Pbl}_{3}$ detectors of $(2.6 \pm 0.4) \times 10^{6} \mu \mathrm{C} \mathrm{Gy}{ }^{-1}$ air $\mathrm{cm}^{-2}$ for 60 $\mathrm{kVp} \mathrm{X}$-ray and the lowest detectable dose rate reaches $(5.0 \pm 0.7) \mathrm{nGy} \mathrm{s}^{-1}$, which enables reduced radiation dose to patients in medical $\mathrm{X}$-ray diagnostics.

\footnotetext{
${ }^{1}$ Department of Applied Physical Sciences, University of North Carolina, Chapel Hill, NC 27599, USA. ${ }^{2}$ Department of Mechanical and Materials Engineering, University of Nebraska-Lincoln, Lincoln, NE 68588, USA.『email: jhuang@unc.edu
} 
M etal halide perovskite (MHP) has become a rising star semiconductor candidate for ionizing radiation detection, due to its superior and unique optoelectronic properties, such as large carrier mobility, long carrier recombination lifetime $e^{1,2}$, high defect tolerance ${ }^{3}$, and high stopping power to ionization radiation ${ }^{4-6}$. Perovskite single crystals are an important set of materials not only as the platform for the study of their intrinsic physical properties ${ }^{7}$, but also to achieve record-performance devices such as solar cells ${ }^{8}$, photodetector ${ }^{9}$, and ionization radiation detectors ${ }^{6,10}$. Single crystals have shown better physical properties than polycrystalline materials such as larger mobility, longer recombination lifetime, less ion diffusion $^{11}$, less un-intentional doping ${ }^{12}$, and better stability ${ }^{13}$, which are contributed to the absence of extended defects such as grain boundaries as well as the lower point defect density ${ }^{14}$. To grow MHP single crystals, solution-based methods are still simplest, and low-cost in making perovskite single crystals of different compositions with large quantity. Many efforts have been devoted to grow perovskite single crystals of different compositions and shapes to optimize their device performance. The growth rates of different facets were tailored by choosing the nonstoichiometry of raw material or different solvents, which resulted in the crystals in different shapes ${ }^{15-17}$. Thin crystals with a large aspect ratio were grown on solution/air interface ${ }^{18-20}$ or between two substrates with space confinements ${ }^{21,22}$. All solution-based single crystal growth methods create supersaturated solution so that solute can precipitate out. Among them, crystal growth through inverse temperature crystallization (ITC) method is particularly attractive, which utilizes the reduced solubility of perovskites in different solutions at a range of higher temperature ${ }^{23-25}$. To obtain large-sized bulk single crystals, the single crystal was carefully transferred as seed to another fresh precursor for multiple cycles ${ }^{26}$ or using oil to continuously extract solvents ${ }^{27}$. However, there are much less efforts devoted to enhancing the quality of perovskite single crystals in terms of reducing point defect density, despite it is well known in the community that the crystals quality is important to achieve the best properties and device performance.

In this work, we introduced coordinating organic molecules in perovskite precursor solution as additives to regulate the growth of perovskite single crystals which impact both nucleation and crystal growth processes. The additives with strong interaction to lead ions in perovskites can tune the growth rate of different facets, and more importantly reduce the point defect formation. The obtained single crystals with improved quality showed a smaller defect density by 23 folds, which dramatically suppressed ion migration and improved optoelectronic properties. The application of these high quality $\mathrm{CH}_{3} \mathrm{NH}_{3} \mathrm{PbI}_{3}\left(\mathrm{MAPbI}_{3}\right)$ single crystals for medical X-ray detection achieved the record high sensitivity of $(2.6 \pm 0.4) \times 10^{6} \mu \mathrm{C} \mathrm{Gy}^{-1}$ air $\mathrm{cm}^{-2}$ and lowest detectable dose rate of $(5.0 \pm 0.7) \mathrm{nGy} \mathrm{s}^{-1}$ for $60 \mathrm{kVp} \mathrm{X}$-ray.

\section{Results and discussion}

Additive modification of crystal growth. Like any crystal growth process, there is a trade-off between growth speed and crystal quality. A higher growth temperature is beneficial to reduce the crystal defect density, because the precursor ions have a larger diffusion rate so that they can find the right lattice position to stick to. There are always chances that point defects can be generated due to the absence of an ion or a right type of ion showing up at the lattice position, which may cause the formation of point defects like vacancies or antisites, even at high temperature. Reducing crystal growth speed to decrease defect formation is not always an option in applications. Our method of changing crystal growth kinetics and reducing defect density is to introduce some organic molecules which have strong interaction with ions on the surface of perovskite crystals to regulate the addition of precursor ions onto the crystals. Such molecules should have interaction with ions of perovskites to some extent, but the interaction should not be too strong to form primary chemical bonding permanently, which would hinder or terminate crystal growth. This design rule indicates that most organic molecules which are broadly used for defect passivation in perovskite solar cells can act on this role. Here we chose one molecule previously studied for surface passivation on MHP thin film solar cells, 3-(Decyldimethylammonio)-propane-sulfonate inner salt (DPSI), because it not only passivates defects in MHP polycrystalline grains, but also modulates the grain size in the polycrystalline films ${ }^{28}$. The $\mathrm{MAPbI}_{3}$ single crystals were grown using the ITC method where crystals precipitate from $\mathrm{MAPbI}_{3}$ precursor solutions after they were heated to a growth temperature which is dependent on the precursor concentration ${ }^{25,29}$. The $\mathrm{MAPbI}_{3}$ single crystal grown from control precursor solution without DPSI was dodecahedral shape, and exposed facets are (100)t and (112)t, where $t$ donates tetragonal phase, as shown in Fig. 1a. After adding $10 \%$ molar ratio of DPSI to $\mathrm{Pb}$ in the precursor solution, the grown crystals had more cuboid shape with facets of (002)t, (110)t, and (112)t. A single crystal with dimensions of $12.9 \mathrm{~mm} \times 12.6 \mathrm{~mm} \times 3.7 \mathrm{~mm}$ was obtained from this precursor with $10 \%$ DPSI at $108^{\circ} \mathrm{C}$ after growing for $50 \mathrm{~h}$ (Fig. 1a). Further increasing the DPSI ratio to $16.3 \%$ resulted in disappearance of the higher index plane of (112) and thus completely cuboid shape crystals (Fig. 1a). Here the facets of these crystals were determined by X-ray diffraction (XRD) measurement, as shown in Fig. $1 \mathrm{~b}$ and Supplementary Fig. 1. The powders diffraction pattern of the $\mathrm{MAPbI}_{3}$ cuboid-shape crystal grown with $16.3 \mathrm{~mol} \%$ DPSI shows it is still tetragonal phase (Fig. 1b). It is noted that the required nucleation temperature was $18-20^{\circ} \mathrm{C}$ higher, while the growth temperature only needed $8^{\circ} \mathrm{C}$ higher after pre-seeding to reach the same growth rate (Supplementary Fig. 2). To test whether this method is general in controlling crystal growth, DPSI was then added into the single crystal growth of a different composition of $\mathrm{MAPbBr}_{3}$. As shown in Supplementary Fig. 3a, b, DPSI also reduced the growth rate of $\mathrm{MAPbBr}_{3}$, and a higher growth temperature was required for crystal growth with same rate.

Mechanism of crystal growth using DPSI additive. To understand the interaction of DPSI with perovskites, the Fourier transform infrared spectroscopy (FTIR) measurements were conducted after mixing $\mathrm{MAPb}_{3}$ and DPSI. In Fig. 2a, for pristine DPSI powder (after vacuum drying), the peaks of the sulfonic acid group at $1030 \mathrm{~cm}^{-1}$, $1039 \mathrm{~cm}^{-1}$ were observed ${ }^{30,31}$. The two peaks are assigned as $\mathrm{S}^{-} \mathrm{O}^{-}$ $\left(1030 \mathrm{~cm}^{-1}\right)$ and $\mathrm{S}=\mathrm{O}\left(1039 \mathrm{~cm}^{-1}\right)$ in ${ }^{-\mathrm{SO}_{3}}{ }^{-}$group of DPSI. To further understand the interaction between DPSI and $\mathrm{MAPbI}_{3}$, FTIR characterization was also applied onto the mixtures of the $\mathrm{PbI}_{2}: \mathrm{DPSI}$, MAI:DPSI and $\mathrm{MAPbI}_{3}: \mathrm{DPSI}$, respectively, which were prepared by dissolving them in solvents and drying the solution. The peak shifts of $-\mathrm{SO}_{3}{ }^{-}$group in FTIR spectra of the three samples were compared with that of pristine DPSI powder. A fitted peak of $1047 \mathrm{~cm}^{-1}$ in MAI:DPSI sample suggests that $\mathrm{MA}^{+}$forms hydrogen bond with $\mathrm{S}=\mathrm{O}$ in DPSI. For $\mathrm{PbI}_{2}:$ DPSI sample, the $\mathrm{S}^{-} \mathrm{O}^{-}$peak of DPSI redshifted, and it can be fitted into two new peaks of $1018 \mathrm{~cm}^{-1}$ and $1024 \mathrm{~cm}^{-1}$, which indicates there are more than one interaction scenarios of $\mathrm{Pb}^{2+}$ with $\mathrm{S}-\mathrm{O}$. The shifting of $\mathrm{S}=\mathrm{O}$ from $1039 \mathrm{~cm}^{-1}$ to $1054 \mathrm{~cm}^{-1}$ suggests a coordination interaction of the lone pair electrons of oxygen in $\mathrm{S}=\mathrm{O}$ with $\mathrm{Pb}$. The $\mathrm{S}^{-} \mathrm{O}^{-}$peak in $\mathrm{MAPbI}_{3}$ : DPSI is dominantly by the red-shifted $1030 \mathrm{~cm}^{-1}$ peak, and the main fitted peaks are still $1018 \mathrm{~cm}^{-1}$ and $1024 \mathrm{~cm}^{-1}$, which are caused by the interaction of $\mathrm{S}^{-} \mathrm{O}^{-}$with $\mathrm{Pb}^{2+}$. The hydrogen bonds of 
a

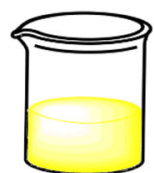

$\mathrm{MAPbI}_{3}$ precursor

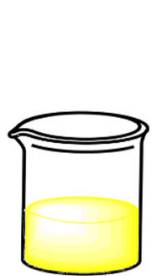

$\mathrm{MAPbI}_{3}$

precursor
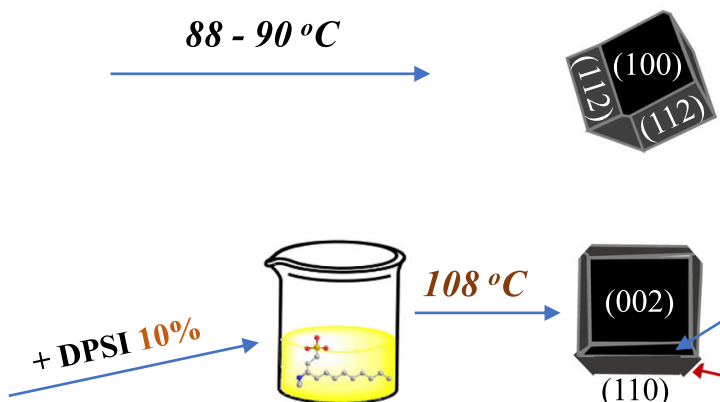

(112)

$(100)$

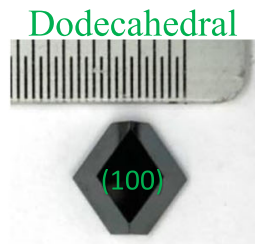

Diamond-shape

b

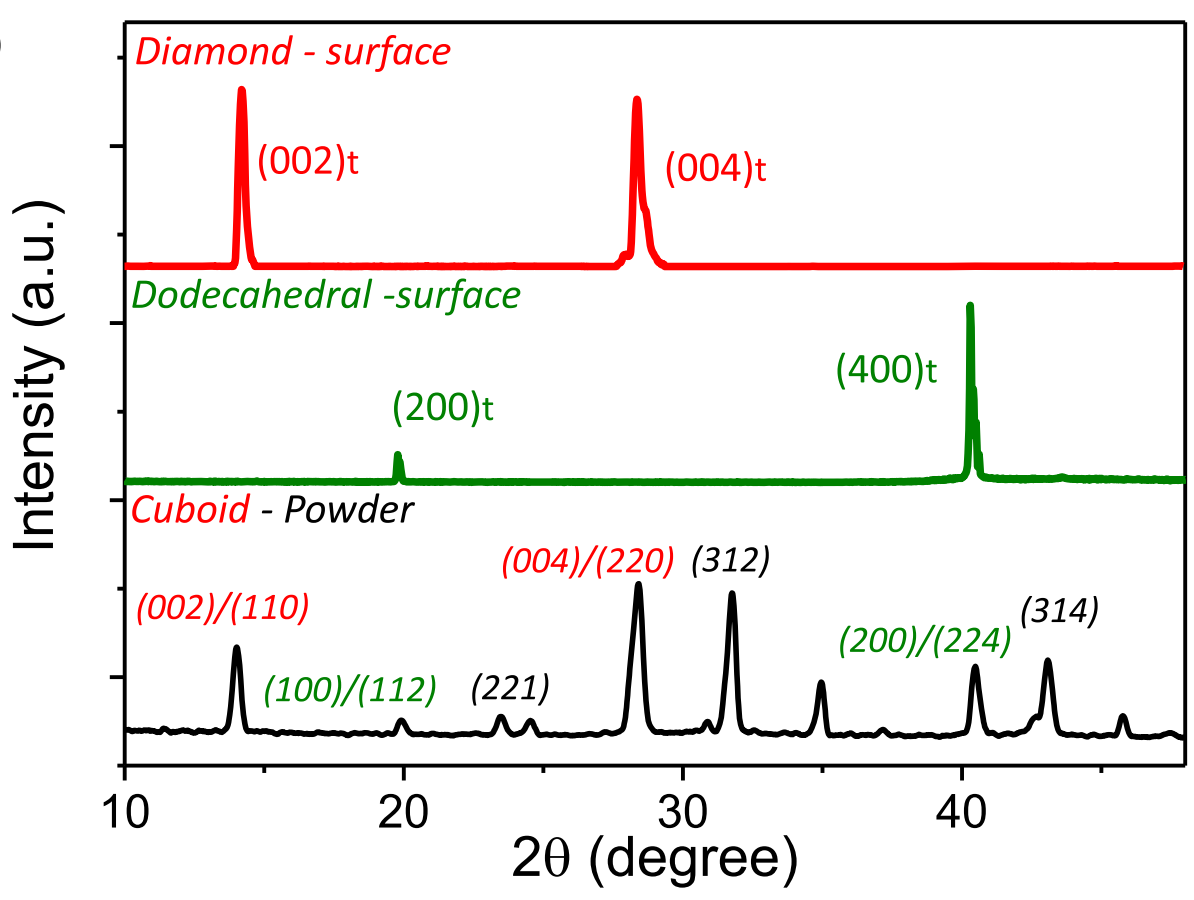

Fig. 1 Crystal growth behavior with ligand regulation. a Scheme of crystals growing process and conditions, and photographs of the grown crystals. $\mathbf{b} X \mathrm{RD}$ patterns of the facets of the crystals and powder of the cuboid-shape crystal.

$\mathrm{S}=\mathrm{O}$ in DPSI with MA are almost negligible and the ionic bonding of $-\mathrm{SO}_{3}{ }^{-}$with $\mathrm{Pb}^{2+}$ dominates the interaction of DPSI and $\mathrm{MAPbI}_{3}$, as illustrated in Fig. $2 b$.

The strong interaction of the $\mathrm{S}^{-} \mathrm{O}^{-}$group of DPSI with $\mathrm{Pb}^{2+}$ indicates that the surfaces of perovskite crystals in solution can be wrapped by DPSI. The steric hindrance effect from the long $\left(\mathrm{CH}_{2}\right)_{n}$ - chain of the DPSI, working as the polymer brushes with a thickness of 1-2 $\mathrm{nm}^{32}$, would hinder the diffusion of ions to the perovskite surfaces for attachment, as illustrated in Fig. 2c. The hindrance effect of DPSI would change the growth rate of different facets ${ }^{33,34}$. We calculated the $\mathrm{Pb}$ atom density at different facets. As shown in Table $1, \mathrm{~Pb}$ atom density on (110)t and $(002) t$ is largest, and thus these facet would grow slower and survive. The suppression of crystal growth at (110)t and (002)t facets were directly observed when DPSI was added with different weight percentages into the precursor solution after seeding with regular dodecahedral-shaped crystals (Supplementary Fig. 2a).
The (112) facet with a lower density of $\mathrm{Pb}$ ions would grow much faster, eventually resulting in its disappearance (Fig. 1a and Supplementary Fig. 2a $)^{35}$ To maintain the same crystal growth rate, the crystal growth temperature needed to be increased from $88^{\circ} \mathrm{C}$ to $96^{\circ} \mathrm{C}$, as shown in Supplementary Fig. 2 b. We speculate that an elevated temperature would also enhance the dynamic detaching of DPSI to the perovskite surfaces, which allows more solute ions to diffuse and attach to crystal surfaces, as illustrated in Fig. 2c. The increased crystal growth temperature also enhanced the diffusion rate of ions in the precursor solution, which allows the right ions to be delivered timely to the surface lattice position and thus reduces the defect density in the grown single crystals ${ }^{36,37}$. This could not be achieved by simply enhancing the crystal growth temperature in regular precursors without DPSI, because an increased temperature generally causes the formation of a larger quantity of nucleus everywhere in the solution. A too large density of nucleus in solution would quickly 
a

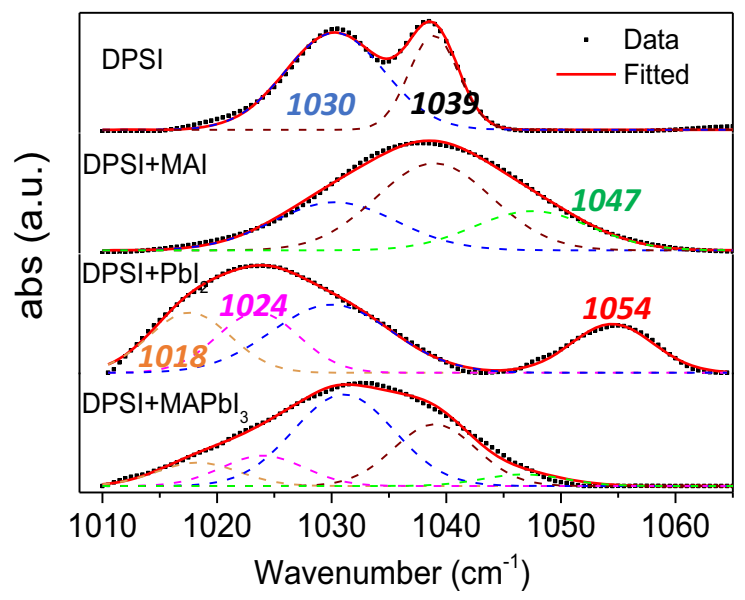

C

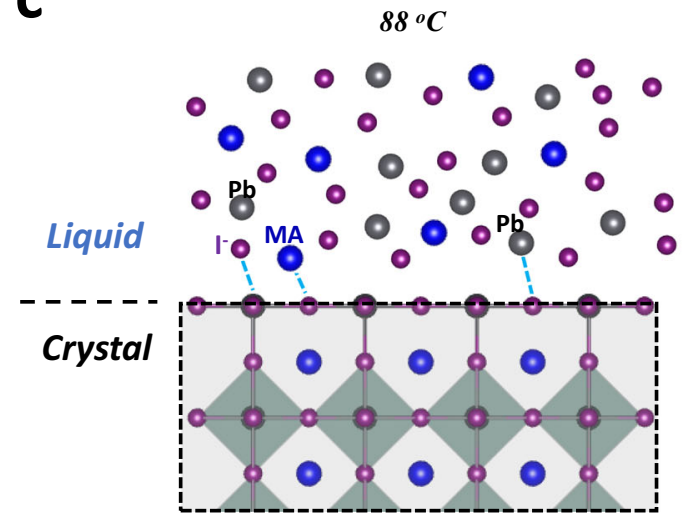

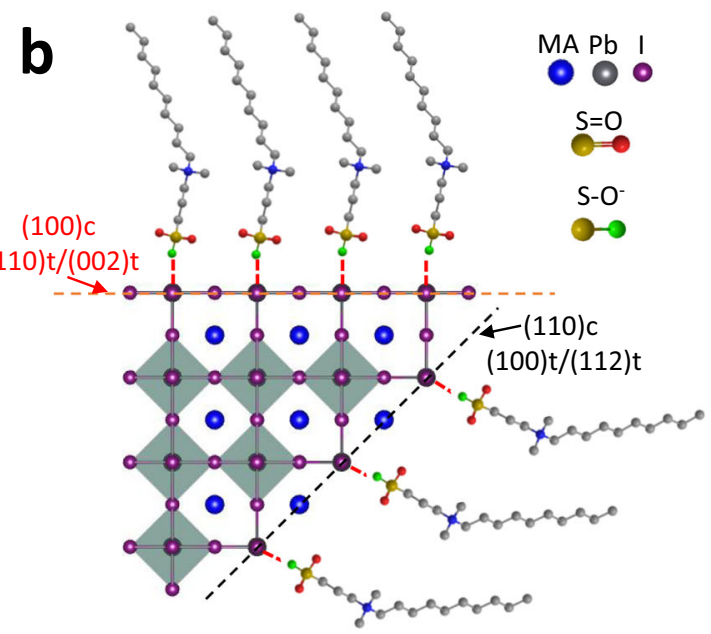

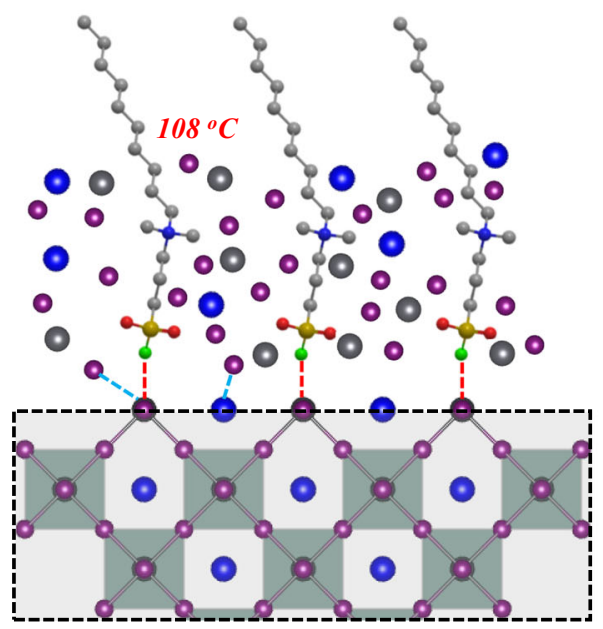

Fig. 2 Mechanism of crystal growth with DPSI. a FTIR spectra of the powders of MAI, Pbl 2 , and MAPbl 3 with 10 mol\% DPSI. b Scheme of DPSI absorption difference on different crystal facets which have different $\mathrm{Pb}^{2+}$ density. c Scheme of crystal growth mechanism at the surface of crystal where the DPSI molecules modulate the diffusion of metal ions to reach the perovskite surface.

\section{Table 1 Calculated atomic area density in different facets of} typical $\mathrm{MAPbl}_{3}$ crystals in tetragonal phase.

\begin{tabular}{lll} 
Facets & $\mathbf{P b}$ atomic density (atoms $\AA^{-\mathbf{2}}$ ) & $\mathbf{I}$ atomic density (atoms $\AA^{-2}$ ) \\
\hline$\{002\} \mathrm{t}$ & 0.02548 & 0.05096 \\
$\{110\} \mathrm{t}$ & 0.02522 & 0.05044 \\
$\{100\} \mathrm{t}$ & 0.01824 & 0.01824 \\
$\{112\} \mathrm{t}$ & 0.01793 & 0.01793 \\
\hline
\end{tabular}

consume the solute in the solution, and they would attach to other growing crystals, which prevents the crystals growing into large size, as demonstrated by the growth of $\mathrm{MAPbBr}_{3}$ in Supplementary Fig. 3a. Therefore, another main function of DPSI is to suppress the nucleation, as evidenced by the less crystals in the solution with DPSI in Supplementary Fig. 3). Since the temperature needs to be $20^{\circ} \mathrm{C}$ (for $\mathrm{MAPbI}_{3}$ with DPSI) higher than normal to reach the same nucleation rate, while the same crystal growth rate can be achieved by increasing temperature by only $8{ }^{\circ} \mathrm{C}$. DPSI additive enables a new crystal growth temperature window to achieve the same growth rate but with suppressed nucleation. Finally, the interaction of $\mathrm{Pb}^{2+}$ or undercoordinated- $\mathrm{Pb}$ with DPSI also introduces an energy barrier for the diffusing ions for further crystal growth. Only if the binding energy of incoming species with $\mathrm{Pb}$ is stronger than
$\mathrm{Pb}$-DPSI under high growth temperature, such as $\mathrm{I}^{-} \mathrm{Pb}$ bonding, DPSI would be replaced and the crystal can keep growing. This mechanism can effectively increase the diffusion barrier and the defect formation energy to reduce the possibility of bonding or stacking of wrong ions.

High quality single crystals. The changed crystal growth kinetics of $\mathrm{MAPbI}_{3}$ single crystal grown with DPSI additive should also affect the crystal crystallinity. To investigate whether DPSI introduces a crystallinity difference, we conducted X-ray rocking curve measurements on these crystals. Here nine samples from each type of crystals were studied for a statistical comparison. The photographs of some crystals with DPSI in different size are shown in Supplementary Fig. 4. As shown in Fig. 3a, the average full width at half maximum (FWHM) of the rocking curve for (002) facet was $0.0289^{\circ}$ for the crystals grown with DPSI, while that value was $0.1650^{\circ}$ for the crystals grown without additives, and its distribution is also much broader (insert of Fig. 3a). This small FWHM of $0.0199^{\circ}$ for the champion crystal grown with DPSI almost reached the resolution of our equipment, and is among the best quality $\mathrm{MAPbI}_{3}$ single crystals reported so far. Such a sharp rocking curve for the $\mathrm{MAPbI}_{3}$ single crystals grown with DPSI confirmed that the crystallinity has been dramatically improved. Normally the crystal with improved crystallinity will has a lower defect density. We then measured the charge trap 

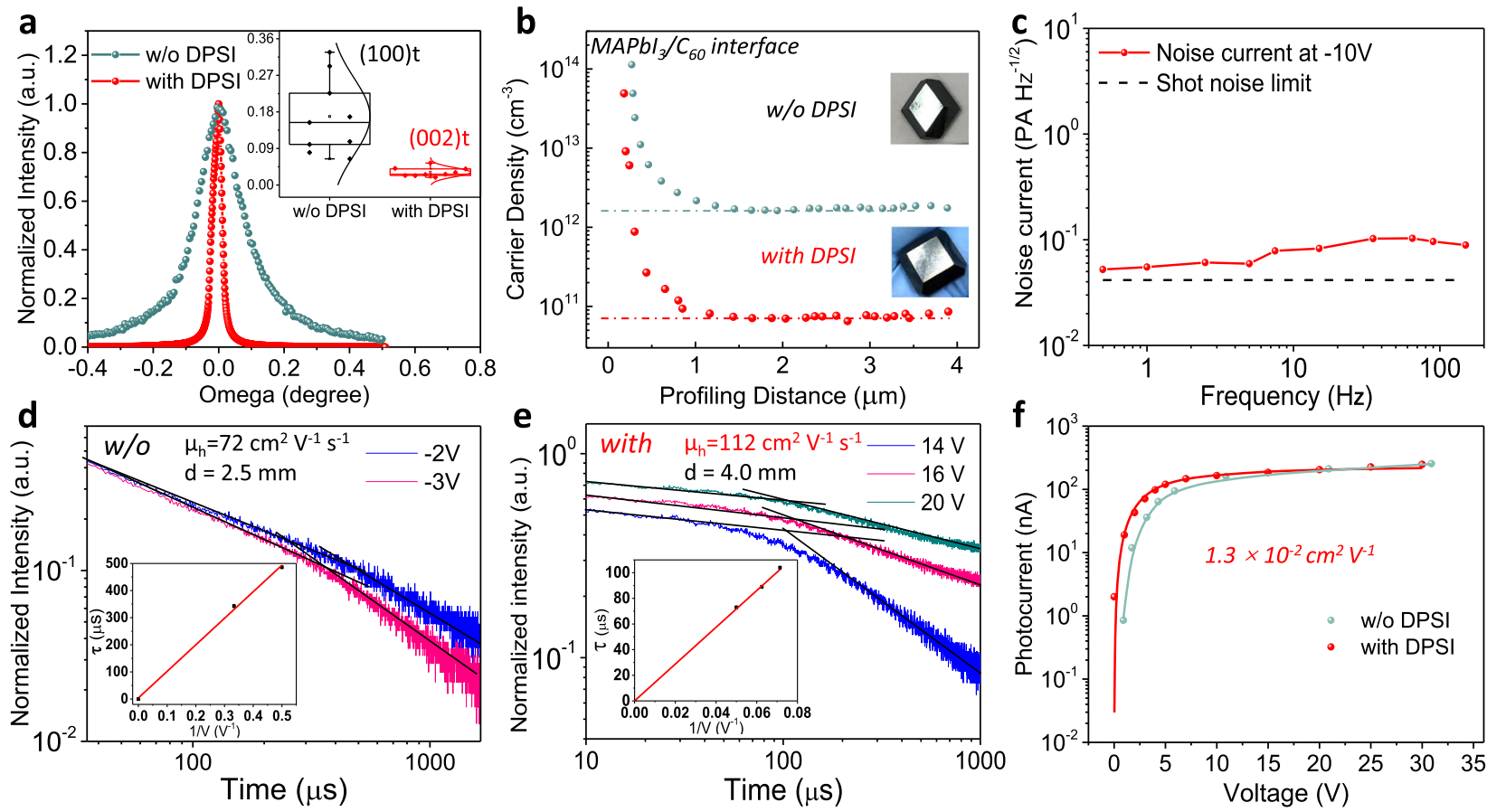

Fig. 3 Material and electronic characterization of $\mathbf{M A P b l}_{\mathbf{3}}$ crystals. a X-ray rocking curves for MAPbl 3 single crystals grown w/o and with DPSI, and the statistical FWHM results are shown as the insert. b Trap density profiled by DLCP for the crystals at different profiling distance from top surface. c Noise current of the crystal grown with DPSI. d, e Time of flight transient currents of the crystals grown w/o and with DPSI. f Photoconductivity comparison of the crystal $\mathrm{w} / \mathrm{o}$ and with DPSI under weak ambient light intensity of $1 \times 10^{-4} \mathrm{~W} \mathrm{~cm}^{-2}$.

density caused by defects using drive-level capacitance profile (DLCP) technique, which was recently shown to characterize the spatial distribution of defect densities in perovskite single crystals ${ }^{14}$. Figure $3 \mathrm{~b}$ shows the depth dependent trap densities for deep traps with a depth of $>0.43 \mathrm{eV}$ from the top surface $\left(\mathrm{C}_{60} /\right.$ $\mathrm{MAPbI}_{3}$ interface) to the interior for the as-grown crystals without any surface treatment. It is noted these crystals still have a large density of defects at very top surfaces, because there was some precursor residual on the crystal surfaces when the crystals were removed out of precursor solution. The trap density at the interior of $\mathrm{MAPbI}_{3}$ crystals grown with DPSI reached $7 \times 10^{10} \mathrm{~cm}^{-3}$, which is the smallest directly measured trap density among all perovskite single crystals reported in literature. The deep trap density in the $\mathrm{MAPbI}_{3}$ crystals grown with DPSI is about 23 times smaller than the control $\mathrm{MAPbI}_{3}$ crystal, directly proves that DPSI additive can reduce the defect density in the single crystals. This shows that high-quality single crystals with reproducible good crystallinity can be obtained without sacrificing the growth rate. The presence of charge traps would generally cause a frequency dependent noise (pink or $1 /$ f noise with a high noise a low frequency) due to the charge trapping and detrapping process ${ }^{38-40}$. To evaluate the impact of the reduced defect trap density on the device noise, we directly measured it by a Fast Fourier Transform (FFT) signal analyzer and a low noise current preamplifier within the frequency range from $0.5 \mathrm{~Hz}$ to $150 \mathrm{~Hz}$ at bias of $-10 \mathrm{~V}$. Figure $3 \mathrm{c}$ shows that the noise current of the crystal grown with DPSI was almost independent of frequency, which again supports the very low defect density in the crystal.

The hole carrier mobility of the $\mathrm{MAPbI}_{3}$ crystals was also compared by time of flight (TOF) method. As shown in Fig. 3d, $\mathrm{e}$, the hole carrier mobility in $\mathrm{MAPbI}_{3}$ crystal grown with DPSI was largely improved from $72 \mathrm{~cm}^{2} \mathrm{~V}^{-1} \mathrm{~s}^{-1}$ to $112 \mathrm{~cm}^{2} \mathrm{~V}^{-1} \mathrm{~s}^{-1}$. We also compared the $\mathrm{MAPbBr}_{3}$ single crystals to see if DPSI can also improve crystal quality. As shown in Supplementary
Fig. 5, the hole mobility of the MAPbBr $r_{3}$ crystal with DPSI was slightly increased from $132 \mathrm{~cm}^{2} \mathrm{~V}^{-1} \mathrm{~s}^{-1}$ to $144 \mathrm{~cm}^{2} \mathrm{~V}^{-1} \mathrm{~s}^{-1}$, while the electron carrier mobility increased dramatically from $65 \mathrm{~cm}^{2} \mathrm{~V}^{-1} \mathrm{~s}^{-1}$ to $224 \mathrm{~cm}^{2} \mathrm{~V}^{-1} \mathrm{~s}^{-1}$. The carrier mobility-lifetime product $(\mu \tau)$ was derived by fitting the photocurrent of the $\mathrm{MAPbI}_{3}$ devices with modified Hecht equation. As shown in Fig. 3f, the $\mu \tau$ of $\mathrm{MAPbI}_{3}$ crystal was enhanced from $8.4 \times 10^{-3} \mathrm{~cm}^{2} \mathrm{~V}^{-1}$ to $1.3 \times 10^{-2} \mathrm{~cm}^{2} \mathrm{~V}^{-1}$ after growth with DPSI under ambient light with an intensity of $1 \times$ $10^{-4} \mathrm{~W} \mathrm{~cm}{ }^{-2}$, one of the highest reported values for MHPs.

It is noted that not all defects cause electronic charge traps, and some structural defects may cause doping or facilitate ion migration 12,14 . The iodine-based MHP materials are promising candidates in X-ray detection, due to the high atomic number, large attenuation coefficient, and high photogenerated carrier density ${ }^{6,41}$. However, the most widely studied MHPs for X-ray radiation detectors were mainly bromine-based compositions for their low noise level ${ }^{42-45}$. Iodine-based perovskites were reported to have low resistivity and obvious ion migration under bias ${ }^{6,46}$. Ion migration-related current drift or polarization effect has been a major concern for the application of MHPs for radiation detection. It is noted that the fastest ion migration occurs through point defects or extended defects. We thus speculate the perovskite single crystals with a much low defect density should be much more stable under electric field. To evaluate the stability and quality improvement of the single crystal by adding DPSI, we applied a bias for an elongated time to track the dark current drift ${ }^{43}$, since dark current drift has been shown to be a good indicator of ion migration or polarization effect in many type of single crystal materials ${ }^{47,48}$. The as-grown $\mathrm{MAPbI}_{3}$ single crystals with DPSI have been fabricated into detector devices with guard ring ${ }^{42}$ and the structure of $\mathrm{Cr}(20 \mathrm{~nm}) / \mathrm{MAPbI}_{3} / \mathrm{C}_{60}(30 \mathrm{~nm}) /$ bathocuproine $(B C P, 8 \mathrm{~nm}) / \mathrm{Cr}(20 \mathrm{~nm})$. As shown in Supplementary Fig. 6 
a

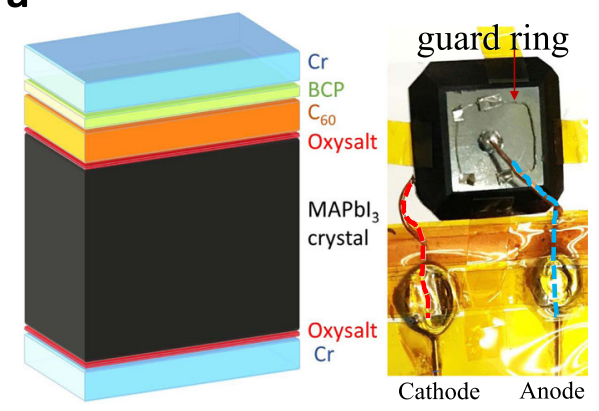

C

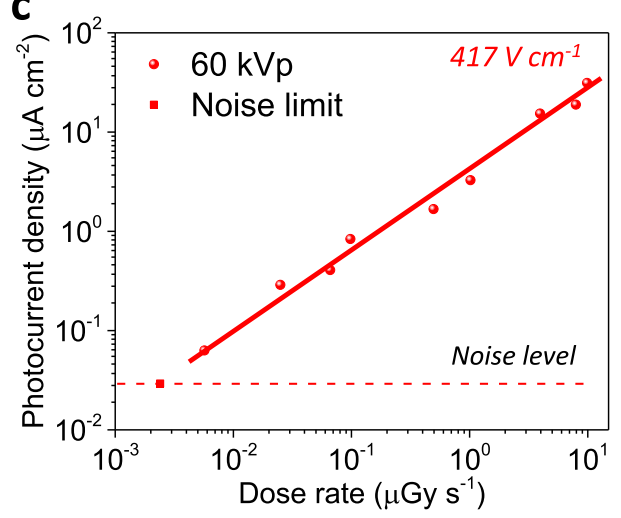

b

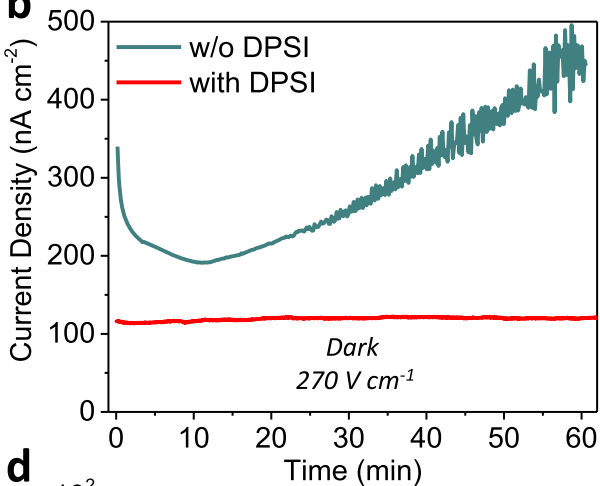

d

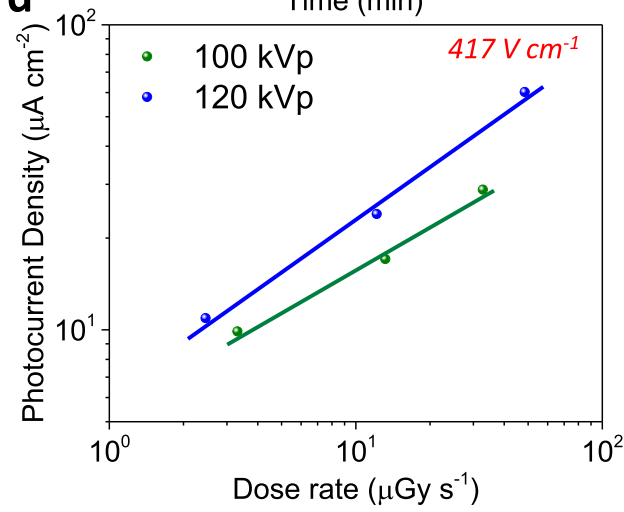

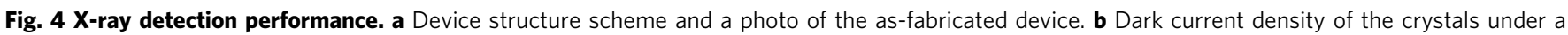
same electric field of $270 \mathrm{~V} \mathrm{~cm}^{-1}$ at ambient air without encapsulation. The crystals were both polished and passivated by oxysalt. The thickness of the

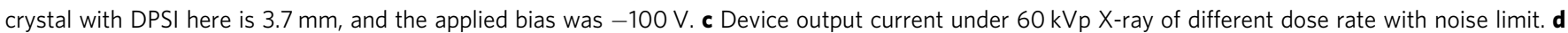
Device output current under $100 \mathrm{kVp}$ and $120 \mathrm{kVp}$ X-rays. This crystal thickness is $2.4 \mathrm{~mm}$, and the applied bias was $-100 \mathrm{~V}$.

and Supplementary Table 1, the dark current drift of these high-quality crystals without any surface treatment or passivation under different electric field reached $3.72 \pm 2.23 \times 10^{-5} \mathrm{nA}$ $\mathrm{cm}^{-1} \mathrm{v}^{-1} \mathrm{~s}^{-1}$, which represents the lowest dark-current drift for iodine-based MHP devices. Since surface defects still exist at the perovskite crystal surface, as suggested by DLCP results, we further polished all $\mathrm{MAPbI}_{3}$ single crystals to remove the surface residuals and treated the exposed surface with $\left(\mathrm{C}_{8} \mathrm{H}_{17} \mathrm{NH}_{3}\right)_{2} \mathrm{SO}_{4}$ solution for $\sim 2 \mathrm{~s}$ at room temperature. And then the crystals were annealed at $50{ }^{\circ} \mathrm{C}$ for $5 \mathrm{~min}$, which induces the formation of a robust, wide bandgap oxysalt to passivate the crystal surface dangling bonds ${ }^{49}$. These treatments can slow down the surface degradation under bias which would otherwise cause electrochemical reaction of perovskite with metal electrodes. Device structure of the crystals after surface treatments is shown in Fig. 4a. As shown in Fig. 4b, the crystal grown with DPSI is not only more intrinsic due to the smaller dark current of $\sim 48 \mathrm{nA}$ (dark current density of $120 \mathrm{nA} \mathrm{cm}^{-2}$ ) under a field of $270 \mathrm{~V} \mathrm{~cm}^{-1}$, but also shows more than 10 times less dark current drift than pristine crystal during $1 \mathrm{~h}$ continuous test (Fig. 4a). The dark current density drift of the $\mathrm{MAPbI}_{3}$ crystal device with DPSI under such a high bias was $1.2 \times 10^{-5} \mathrm{nA} \mathrm{cm}^{-1} \mathrm{v}^{-1} \mathrm{~s}^{-1}$ (Table 2), which is comparable to the best reported inorganic perovskites $\mathrm{Cs}_{2} \mathrm{AgBiBr}_{6}$ device ${ }^{43}$. It is known that $\mathrm{I}^{-}$ions are much more mobile than $\mathrm{Br}^{-}$ions ${ }^{50}$, therefore the small dark current drift in $\mathrm{MAPbI}_{3}$ crystal devices indicates a significant smaller iodine-related defect density. The resistivity of the $\mathrm{MAPbI}_{3}$ crystal detector with DPSI under different electric field was measured to be $1.95 \pm 0.35 \times 10^{9} \Omega \mathrm{cm}$ (Table 2), which is among the highest of iodide-based perovskite and also suggests their low defect density, since defect can cause self-doping $14,51,52$.
Table 2 Dark current drift and resistivity result of the $\mathrm{MAPbl}_{3}$ crystal with DPSI after surface treatment in Fig. 4b.

\begin{tabular}{|c|c|c|c|c|}
\hline $\begin{array}{l}\text { Reverse } \\
\text { bias (V) }\end{array}$ & $\begin{array}{l}\text { Electric field } \\
\left(\mathrm{V} \mathbf{~ c m}^{-1}\right)\end{array}$ & $\begin{array}{l}\text { Dark current } \\
\left(\mathrm{nA} \mathbf{c m}^{-2}\right)\end{array}$ & $\begin{array}{l}\text { Resistivity ( } \Omega \\
\text { cm) }\end{array}$ & $\begin{array}{l}\text { Current drift (nA } \\
\left.\mathrm{cm}^{-1} v^{-1} s^{-1}\right)\end{array}$ \\
\hline $\begin{array}{l}5 \\
10 \\
20 \\
100\end{array}$ & $\begin{array}{l}13.5 \\
27 \\
54 \\
270\end{array}$ & $\begin{array}{r}8.7 \\
15.6 \\
34.5 \\
120\end{array}$ & $\begin{array}{l}1.55 \times 10^{9} \\
1.73 \times 10^{9} \\
1.56 \times 10^{9} \\
2.25 \times 10^{9}\end{array}$ & $\begin{array}{r}1.6 \times 10^{-6} \\
6.58 \times 10^{-6} \\
1.3 \times 10^{-5} \\
1.2 \times 10^{-5}\end{array}$ \\
\hline
\end{tabular}

Application of crystals for X-ray detection. Finally, we measured the X-ray detection performance using these high-quality $\mathrm{MAPbI}_{3}$ crystals. Firstly, the X-ray detector based on the crystal of Fig. 4b with DPSI has shown excellent sensitivity of $2.1 \times 10^{5} \mu \mathrm{C} \mathrm{Gy}^{-1}$ air $\mathrm{cm}^{-2}$ with lowest detectable dose rate of $2.34 \mathrm{nGy} \mathrm{s}^{-1}$ under soft Xray energy with $8 \mathrm{keV}$ (Supplementary Fig. 7). However, in medical imaging, hard X-rays with energy above $60 \mathrm{kVp}$ are mostly used for a high penetration capability, so the hard X-rays with energy of 60 $\mathrm{kVp}, 100 \mathrm{kVp}$ and $120 \mathrm{kVp}$ were used in this study with their simulated energy spectrum shown in Supplementary Fig. 8. Under bias of $-100 \mathrm{~V}$ on a crystal with thickness of $2.4 \mathrm{~mm}$ (electric field of $417 \mathrm{~V} \mathrm{~cm}^{-1}$ ), the dark current was measured about $81 \mathrm{nA}$ (current density of $2.71 \times 10^{3} \mathrm{nA} \mathrm{cm}^{-2}$ ) with $4.7 \times 10^{-5} \mathrm{nA} \mathrm{cm}^{-1} \mathrm{v}^{-1} \mathrm{~s}^{-1}$ drifted under continuous tracking. This detector delivered high sensitivities of $2.9 \times 10^{6} \mu \mathrm{C} \mathrm{Gy}^{-1}$ air $\mathrm{cm}^{-2}$ for $60 \mathrm{kVp} \mathrm{X}$-ray (Fig. 4c), $6.01 \times 10^{5} \mu \mathrm{C} \mathrm{Gy}^{-1}$ air $\mathrm{cm}^{-2}$ for $100 \mathrm{kVp} \mathrm{X}$-ray, and $1.04 \times 10^{6} \mu \mathrm{C}$ $\mathrm{Gy}^{-1}$ air $\mathrm{cm}^{-2}$ for $120 \mathrm{kVp}$ (Fig. 4d). The lowest detectable dose rate

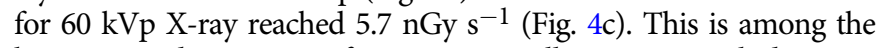
best X-ray detection performances in all MHP crystal detectors regardless of compositions, as summarized in Supplementary Table 2. The low noise of these high quality $\mathrm{MAPbI}_{3}$ single crystals allows them to compete with bromine-based and low-dimension 
MHPs, such as $\mathrm{MAPbBr}_{\mathrm{x}} \mathrm{Cl}_{3-\mathrm{x}}{ }^{42}, \mathrm{Cs}_{2} \mathrm{AgBiBr}_{6}{ }^{43}, \quad\left(\mathrm{NH}_{4}\right)_{3} \mathrm{Bi}_{2} \mathrm{I}_{9}$ (2D crystal) ${ }^{44}$, given that their lower bandgap and higher atomic number of I than $\mathrm{Br}$ should allow the generation of a larger density of charge carriers under X-ray.

In this work, we have significantly improved the quality of $\mathrm{MAPbI}_{3}$ single crystals by ligand regulation during crystal growth, with 23 times lower bulk defect density, without sacrificing crystal growth rate. The ion migration under high bias has been successfully suppressed for the first time for iodide perovskites, which is comparable to inorganic perovskites. The crystal detectors achieve ultra-high sensitivity of $(2.6 \pm$ $0.4) \times 10^{6} \mu \mathrm{C} \mathrm{Gy}^{-1}$ air $\mathrm{cm}^{-2}$ and the lowest detectable dose rate of $5.0 \pm 0.7 \mathrm{nGy} \mathrm{s}^{-1}$ to $60 \mathrm{kVp} \mathrm{X}$-ray.

\section{Methods}

Materials and crystal growth. The solvents of $\gamma$-butyrolactone (GBL, >99\%), N, N-Dimethylformamide (DMF, > 98\%), lead bromide $\left(\mathrm{PbBr}_{2},>98 \%\right)$, BCP (96\%), and DPSI were all purchased from Sigma-Aldrich. MAI and MABr powder were purchased from GreatCell Solar. Lead iodide $\left(\mathrm{PbI}_{2}, 99.9985 \%\right)$ was purchased from Alfa Aesar. All the MHP single crystals were grown by typical ITC method respectively. $\mathrm{C}_{60}(>99.5 \%)$ was purchased from Nano-C and $\mathrm{Cr}$ was purchased from Kurt J. Lesker. For $\mathrm{MAPbI}_{3}$ single crystals in this study, MAI and $\mathrm{PbI}_{2}$ powder were mixed in molar ratio of $1: 1$ with concentration of $1.5 \mathrm{M}$ in GBL to prepare the same initial precursor solution. For $\mathrm{MAPbBr}_{3}$ single crystals, $\mathrm{MABr}$ and $\mathrm{PbBr}_{2}$ powder were mixed in molar ratio of $1: 1$ with concentration of $1.2 \mathrm{M}$ in DMF to prepare the initial precursor. The oxysalt passivation solution was synthesized with the same method ${ }^{49}$ and diluted with the concentration of $1 \mathrm{mM}$ in mixed solvents (toluene/isopropanol $=5: 1$ ).

Characterizations. XRD patterns were carried out on a Rigaku MiniFlex $6 \mathrm{G}$ system with an X-ray tube at $2.0 \mathrm{~kW}$. This was configured with $\mathrm{Cu} \mathrm{Ka}$ radiation (wavelength of $1.5418 \AA$ ). The medium resolution X-ray rocking curve measurements were conducted by a Rigaku Smartlab X-ray diffractometer equipped with a $3 \mathrm{~kW} \mathrm{Cu}$ tube at $\mathrm{V}=40 \mathrm{kV}$ and $\mathrm{I}=44 \mathrm{~mA}$. The parallel beam geometry was applied to achieve medium resolution parallel X-ray source, which is further confined with a $10 \mathrm{~mm}$ slit. The step size for omega scan is 0.0001 degree. The FTIR spectra of the powder samples were scanned from $4000 \mathrm{~cm}^{-1}$ to $400 \mathrm{~cm}^{-1}$ and recorded on a PerkinElmer FTIR spectrometer in reflection mode. All the mixture samples of $\mathrm{PbI}_{2}: \mathrm{DPSI}, \mathrm{MAI}: \mathrm{DPSI}$, and $\mathrm{MAPbI}_{3}: \mathrm{DPSI}$ for FTIR were prepared by drying out their blended solution to study their interactions.

Capacitance measurements. The DLCP measurements were performed by using an Agilent E4980A precision LCR meter. The scanning range of the ac frequency (f) was $0.02-2000 \mathrm{kHz}$. The demarcation energy $E_{\omega}=k T \ln \left(\frac{\omega_{0}}{\omega}\right)$ (where $\omega_{0}$ is the attempt-to-escape angular frequency that equals to $2 \pi v_{0} T^{2}$ is derived from the temperature-dependent $C-f$ measurements. The $v_{0}$ is obtained from the fitting of $\ln \left(\frac{T^{2}}{\omega}\right)=\frac{E_{T}}{k T}-\ln \left(2 \pi v_{0}\right)$ obtained at different $T$. Temperature-dependent capacitance measurements were carried out in a Lake Shore Cryotronics probe stage with a Lake Shore Cryotronics temperature controller model 336. SMA connected cables and ZN50R DC/RF probes that were applicable for RF test up to $1 \mathrm{GHz}$ were equipped with the probe station. Before each measurement, the system was selfcalibrated under open-circuit and short-circuit conditions to compensate for any undesired signal from the instrument.

For the DLCP measurements, the dc bias $(V)$ was scanning from $0 \mathrm{~V}$ to the $V_{\mathrm{OC}}$ (e.g., $1.1 \mathrm{~V}$ ) for the single crystal detectors. While ac bias $(\delta V)$ was ranging from 20 to $200 \mathrm{mV}$. The capacitance measured at each $\delta V$ was recorded and fitted with a polynomial function $C=C_{0}+C_{1} \delta V+C_{2}(\delta V)^{2}+\ldots$ to obtain $C_{0}$ and $C_{1}$. With the determination of $C_{0}$ and $C_{1}$, the total carrier density $(N)$ that includes both free carrier density and trap density at the profiling distance $X$ from the junction barrier is calculated by $N=-\frac{C_{0}^{3}}{2 q \varepsilon A^{2} C_{1}}$, where $q$ is the elementary charge, $\varepsilon$ is the dielectric constant of the semiconductor and $A$ is the active area of the junction. The profiling distance from the junction barrier was calculated by $\varepsilon A / C_{0}$, which was changed by tuning the $V$. For each ac bias, an additional offset dc voltage was applied to keep the maximum forward bias constant. The trap density within a certain trap depth range was calculated by subtracting the total carrier density measured at a larger $E_{\omega}$ (lower ac frequency) with that measured at a smaller $E_{\omega}$ (higher ac frequency).

Time of flight (TOF) measurement. ToF measurement was conducted by illuminating the devices with $4 \mathrm{~ns}$ width, $337 \mathrm{~nm}$ laser pulses (SRS N2 laser) from the semitransparent electrodes side. The pulse laser-generated photocurrent was recorded using an Agilent $1 \mathrm{GHz}$ digital oscilloscope (Agilent DSO-X $3104 \mathrm{~A}$ ).

$\mathrm{X}$-ray radiation detection measurement. The continuous $\mathrm{X}$-ray source for $60 \mathrm{kVp}, 100 \mathrm{kVp}$ and $120 \mathrm{kVp}$ energy detection measurements was manufactured by YXLON with a 21-mm Al sheet filter and focal spot of $1 \mathrm{~mm}$. The different current of the X-ray source and different thickness of 1100 Aluminum alloy sheets were applied to tune the dose rate values during measurement. The dose rate calibration above $60 \mathrm{nGy} \mathrm{s}^{-1}$ was measured by Raysafe X2 Solo dosimeter and using the same calibration process as our previous work ${ }^{41}$. The dose rate calibration below $60 \mathrm{nGy} \mathrm{s}^{-1}$ was measured by SOEKS Quantum radiation detector dosimeter same as our previous method ${ }^{42}$.

Noise current measurement. Noise current was carried out at different frequency by a Fast Fourier Transform (FFT) signal analyzer (Agilent $35670 \mathrm{~A}$ ) which was connected to a low noise current preamplifier.

\section{Data availability}

The data that support the findings of this study are available from the corresponding author upon reasonable request.

Received: 20 October 2020; Accepted: 18 February 2021; Published online: 16 March 2021

\section{References}

1. Dong, Q. et al. Electron-hole diffusion lengths $>175 \mu \mathrm{m}$ in solution-grown $\mathrm{CH}_{3} \mathrm{NH}_{3} \mathrm{PbI}_{3}$ single crystals. Science 347, 967-970 (2015).

2. Stranks, S. D. et al. Electron-hole diffusion lengths exceeding 1 micrometer in an organometal trihalide perovskite absorber. Science 342, 341-344 (2013).

3. Brandt, R. E., Stevanović, V., Ginley, D. S. \& Buonassisi, T. Identifying defecttolerant semiconductors with high minority-carrier lifetimes: beyond hybrid lead halide perovskites. Mrs Commun. 5, 265-275 (2015).

4. Hunter, D. M., Belev, G., Kasap, S. \& Yaffe, M. J. Measured and calculated Kfluorescence effects on the MTF of an amorphous-selenium based CCD x-ray detector. Med. Phys. 39, 608-622 (2012).

5. Zentai, G. et al. In Mercuric iodide and lead iodide $x$-ray detectors for radiographic and fluoroscopic medical imaging, in Medical Imaging 2003: Physics of Medical Imaging. 77-91 (International Society for Optics and Photonics) (2003)

6. Wei, H. \& Huang, J. Halide lead perovskites for ionizing radiation detection Nat. Commun. 10, 1066 (2019).

7. Huang, J., Yuan, Y., Shao, Y. \& Yan, Y. Understanding the physical properties of hybrid perovskites for photovoltaic applications. Nat. Rev. Mater. 2, 17042 (2017).

8. National Renewable Energy Laboratory, Best research-cell efficiencies chart https://www.nrel.gov/pv/cell-efficiency.html (2020).

9. Fang, Y., Dong, Q., Shao, Y., Yuan, Y. \& Huang, J. Highly narrowband perovskite single-crystal photodetectors enabled by surface-charge recombination. Nat. Photonics 9, 679-686 (2015).

10. Kim, Y. C. et al. Printable organometallic perovskite enables large-area, lowdose X-ray imaging. Nature 550, 87-91 (2017).

11. Shao, Y. et al. Grain boundary dominated ion migration in polycrystalline organic-inorganic halide perovskite films. Energy Environ. Sci. 9, 1752-1759 (2016).

12. Xing, J. et al. Ultrafast ion migration in hybrid perovskite polycrystalline thin films under light and suppression in single crystals. Phys. Chem. Chem. Phys. 18, 30484-30490 (2016).

13. Wang, Q. et al. Scaling behavior of moisture-induced grain degradation in polycrystalline hybrid perovskite thin films. Energy Environ. Sci. 10, 516-522 (2017).

14. $\mathrm{Ni}, \mathrm{Z}$. et al. Resolving spatial and energetic distributions of trap states in metal halide perovskite solar cells. Science 367, 1352-1358 (2020).

15. Zhang, Y., Huang, F. \& Mi, Q. Preferential facet growth of methylammonium lead halide single crystals promoted by halide coordination. Chem. Lett. 45, 1030-1032 (2016)

16. Ding, J. et al. Design growth of $\mathrm{MAPbI}_{3}$ single crystal with (220) facets exposed and its superior optoelectronic properties. J. Phys. Chem. Lett. 9, 216-221 (2018)

17. Liu, M. et al. Unveiling solvent-related effect on phase transformations in $\mathrm{CsBr}-\mathrm{PbBr}_{2}$ system: coordination and ratio of precursors. Chem. Mater. 30, 5846-5852 (2018)

18. Zhumekenov, A. A. et al. The role of surface tension in the crystallization of metal halide perovskites. ACS Energy Lett. 2, 1782-1788 (2017).

19. Liu, Y. et al. Fast growth of thin $\mathrm{MAPbI}_{3}$ crystal wafers on aqueous solution surface for efficient lateral-structure perovskite solar cells. Adv. Funct. Mater. 29, 1807707 (2019)

20. Liu, Y. et al. Surface-tension-controlled crystallization for high-quality 2D perovskite single crystals for ultrahigh photodetection. Matter 1, 465-480 (2019). 
21. Chen, Y.-X. et al. General space-confined on-substrate fabrication of thickness-adjustable hybrid perovskite single-crystalline thin films. J. Am. Chem. Soc. 138, 16196-16199 (2016).

22. Chen, Z. et al. Thin single crystal perovskite solar cells to harvest belowbandgap light absorption. Nat. Commun. 8, 1890 (2017).

23. Liu, Y. et al. Low-temperature-gradient crystallization for multi-inch highquality perovskite single crystals for record performance photodetectors. Mater. Today 22, 67-75 (2019).

24. Wang, W. et al. Electronic-Grade High-Quality Perovskite Single Crystals by a Steady Self-Supply Solution Growth for High-Performance X-ray Detectors. Adv. Mater. 32, 2001540 (2020).

25. Saidaminov, M. I. et al. High-quality bulk hybrid perovskite single crystals within minutes by inverse temperature crystallization. Nat. Commun. 6, 7586 (2015).

26. Liu, Y. et al. Two-inch-sized perovskite $\mathrm{CH}_{3} \mathrm{NH}_{3} \mathrm{PbX}_{3}(\mathrm{X}=\mathrm{Cl}, \mathrm{Br}, \mathrm{I})$ crystals: growth and characterization. Adv. Mater. 27, 5176-5183 (2015).

27. Yao, F. et al. Room-temperature liquid diffused separation induced crystallization for high-quality perovskite single crystals. Nat. Commun. 11, 1194 (2020).

28. Zheng, X. et al. Dual functions of crystallization control and defect passivation enabled by sulfonic zwitterions for stable and efficient perovskite solar cells. Adv. Mater. 30, 1803428 (2018).

29. Saidaminov, M. I., Abdelhady, A. L., Maculan, G. \& Bakr, O. M. Retrograde solubility of formamidinium and methylammonium lead halide perovskites enabling rapid single crystal growth. Chem. Commun. 51, 17658-17661 (2015).

30. Gui, Z., Qian, J., An, Q., Xu, H. \& Zhao, Q. Synthesis, characterization and flocculation performance of zwitterionic copolymer of acrylamide and 4vinylpyridine propylsulfobetaine. Eur. Polym. J. 45, 1403-1411 (2009).

31. Viana, R. B., da Silva, A. B. \& Pimentel, A. S. Infrared spectroscopy of anionic, cationic, and zwitterionic surfactants. Advances in physical chemistry 2012 (2012).

32. Krieg, F. et al. Stable ultraconcentrated and ultradilute colloids of $\mathrm{CsPbX}_{3}(\mathrm{X}=$ $\mathrm{Cl}, \mathrm{Br}$ ) nanocrystals using natural lecithin as a capping ligand. J. Am. Chem. Soc. 141, 19839-19849 (2019).

33. Kern, R. \& Dassonville, R. Growth inhibitors and promoters examplified on solution growth of paraffin crystals. J. Cryst. growth 116, 191-203 (1992).

34. Rimer, J. D. et al. Crystal growth inhibitors for the prevention of L-cystine kidney stones through molecular design. Science 330, 337-341 (2010).

35. Gibbs, J. W. On the equilibrium of heterogeneous substances. Am. J. Sci. 3, 441-458 (1878).

36. Perillat-Merceroz, G. et al. In MOCVD growth mechanisms of ZnO nanorods, Journal of Physics: Conference Series. 012034 (IOP Publishing) (2010).

37. Akiyama, M., Kawarada, Y., Ueda, T., Nishi, S. \& Kaminishi, K. Growth of high quality GaAs layers on Si substrates by MOCVD. J. Cryst. Growth 77, 490-497 (1986).

38. Lin, C. et al. Effects of passivation and extraction surface trap density on the 1/ f noise of HgCdTe photoconductive detector. IEEE Photonics Technol. Lett. 9, 232-234 (1997).

39. Carr, J. A. \& Chaudhary, S. The identification, characterization and mitigation of defect states in organic photovoltaic devices: a review and outlook. Energy Environ. Sci. 6, 3414-3438 (2013).

40. Fang, Y. \& Huang, J. Resolving weak light of sub-picowatt per square centimeter by hybrid perovskite photodetectors enabled by noise reduction. Adv. Mater. 27, 2804-2810 (2015).

41. Zhao, J. et al. Perovskite-filled membranes for flexible and large-area directconversion X-ray detector arrays. Nat. Photonics 14, 612-617 (2020).

42. Wei, $\mathrm{H}$. et al. Dopant compensation in alloyed $\mathrm{CH}_{3} \mathrm{NH}_{3} \mathrm{PbBr}_{3-}{ }_{\mathrm{x}} \mathrm{Cl}_{\mathrm{x}}$ perovskite single crystals for gamma-ray spectroscopy. Nat. Mater. 16, 826-833 (2017).

43. Yang, B. et al. Heteroepitaxial passivation of $\mathrm{Cs}_{2} \mathrm{AgBiBr}_{6}$ wafers with suppressed ionic migration for X-ray imaging. Nat. Commun. 10, 1989 (2019).

44. Zhuang, R. et al. Highly sensitive X-ray detector made of layered perovskitelike $\left(\mathrm{NH}_{4}\right)_{3} \mathrm{Bi}_{2} \mathrm{I}_{9}$ single crystal with anisotropic response. Nat. Photonics 13, 602-608 (2019).

45. Pan, W. et al. $\mathrm{Cs}_{2} \mathrm{AgBiBr}_{6}$ single-crystal X-ray detectors with a low detection limit. Nat. photonics 11, 726-732 (2017).

46. Huang, Y. et al. A-site Cation Engineering for Highly Efficient $\mathrm{MAPbI}_{3}$ SingleCrystal X-ray Detector. Angew. Chem. Int. Ed. 58, 17834-17842 (2019).
47. Ponpon, J. \& Amann, M. Current instability and polarization phenomena in lead iodide crystalline detectors. Nucl. Instrum. Methods Phys. Res. Sect. A : Accelerators, Spectrometers, Detect. Associated Equip. 526, 447-454 (2004).

48. Grill, R. et al. Polarization study of defect structure of CdTe radiation detectors. IEEE Trans. Nucl. Sci. 58, 3172-3181 (2011).

49. Yang, S. et al. Stabilizing halide perovskite surfaces for solar cell operation with wide-bandgap lead oxysalts. Science 365, 473-478 (2019).

50. Meggiolaro, D. et al. Iodine chemistry determines the defect tolerance of leadhalide perovskites. Energy Environ. Sci. 11, 702-713 (2018).

51. Wang, Q. et al. Qualifying composition dependent $\mathrm{p}$ and $\mathrm{n}$ self-doping in $\mathrm{CH}_{3} \mathrm{NH}_{3} \mathrm{PbI}_{3}$. Appl. Phys. Lett. 105, 163508 (2014).

52. Deng, Y. et al. Reduced Self-Doping of Perovskites Induced by Short Annealing for Efficient Solar Modules. Joule 4, 1949-1960 (2020).

\section{Acknowledgements}

This work involving material growth, characterization and device fabrication and testing is mainly supported by Center for Hybrid Organic Inorganic Semiconductors for Energy (CHOISE), an Energy Frontier Research Center funded by the Office of Basic Energy Sciences, Office of Science within the US Department of Energy. The radiation detection research is supported by the Department of the Defense, Defense Threat Reduction Agency (DTRA) under award HDTRA1-20-2-0002. The content of the information does not necessarily reflect the position or the policy of the federal government, and no official endorsement should be inferred.

\section{Author contributions}

J.H. and Y.L. conceived the idea and designed experiments. Y.L. prepared the $\mathrm{MAPbI}_{3}$ crystal devices and conducted their optoelectronic characterizations and FTIR measurements. X.Z. prepared the $\mathrm{MAPbBr}_{3}$ crystal devices and conducted their TOF measurement. Y.L. and Y.F. were involved in soft X-ray detection measurement. Y.L. and Y.Z. were involved in hard X-ray detection measurement. Y.F. conducted FFT noise current measurement. Z.N. conducted the DLCP measurement. X.X. conducted the XRD and X-ray rocking curve measurements. Y.L. and S.C. analyzed the FTIR results. J.H. and Y.L. wrote the manuscript and all authors reviewed it.

\section{Competing interests}

The authors declare no competing interests.

\section{Additional information}

Supplementary information The online version contains supplementary material available at https://doi.org/10.1038/s41467-021-21934-6.

Correspondence and requests for materials should be addressed to J.H.

Peer review information Nature Communications thanks Wei Lei, Mingjian Yuan and the other, anonymous, reviewer(s) for their contribution to the peer review of this work.

Reprints and permission information is available at http://www.nature.com/reprints

Publisher's note Springer Nature remains neutral with regard to jurisdictional claims in published maps and institutional affiliations.

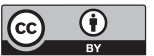

Open Access This article is licensed under a Creative Commons Attribution 4.0 International License, which permits use, sharing, adaptation, distribution and reproduction in any medium or format, as long as you give appropriate credit to the original author(s) and the source, provide a link to the Creative Commons license, and indicate if changes were made. The images or other third party material in this article are included in the article's Creative Commons license, unless indicated otherwise in a credit line to the material. If material is not included in the article's Creative Commons license and your intended use is not permitted by statutory regulation or exceeds the permitted use, you will need to obtain permission directly from the copyright holder. To view a copy of this license, visit http://creativecommons.org/ licenses/by/4.0/.

(C) The Author(s) 2021 